Tohoku J. exp. Med., 1969, 99, 303-30з

\title{
The Microvibration of the Body Surface Related to the Heartbeat in Complete A-V Block
}

\author{
Toshiyuki Ozaki, Katsuro Igarashi, Sechiko Sasaki \\ and Hitomi Takahashi \\ The First Department of Physiology (Prof. T. Ozaki), \\ Hirosaki University School of Medicine, Hirosaki
}

\begin{abstract}
The microvibration (MV) of the thenar was recorded by means of a MV recording technique in normal young adults and patients with complete A-V block. In addition to the MV, the electrocardiogram (EKG) was also traced simultaneously to determine whether the existence of the MV components is related to the pulsation of the heart. The dominant vibrations of the MV were observed to be mainly made up of two definite groups of vibrations corresponding to $R$ and $T$ waves of the EKG in patients with complete A-V block as well as in normal subjects. In complete A-V block, however, the intervals between the dominant vibrations of each group prolonged according to the marked lengthening of the R-R interval in the EKG. Furthermore, the correspondence between the MV and $P$ waves in the EKG wis also observed slightly in complete A-V block. The above-mentioned results show that the dominant vibrations of the MV over the human body surface mainly consist of ballistocardiographic components at rest.
\end{abstract}

It has been described by Inanaga, ${ }^{1}$ and Sugano and Inanaga ${ }^{2}$ that the microvibration (MV) of the human and animal body surface is not related to body vibrations due to the pulsation of the heart, but to the spinal reflex, especially through the gamma motor system. In the previous studies, ${ }^{3-5}$ on the other hand, the present authors revealed some intimate correlations between the dominant vibrations of the MV and the first and second components of the heartbeat, $R$ and $\mathrm{T}$ waves of the electrocardiogram (EKG), or $\mathrm{I}, \mathrm{J}$ and $\mathrm{K}$, and $\mathrm{L}, \mathrm{M}$ and $\mathrm{N}$ waves of the ballistocardiogram (BCG) in man. Buskirk and Fink ${ }^{6}$ considered that this vibration might be ballistocardiographic one. This was confirmed by Yap and Boshes. ${ }^{7}$ These findings suggest that the accelerative and inhibitory effects of the cardiac activity may also play an important role in the augmentation and depression of the MV, although it was denied by Inanaga ${ }^{1}$ and Yoshii et al. ${ }^{8}$ Accordingly, it seems very interesting to examine the MV under various conditions which affect the heart rate. The present experiments were undertaken in an attempt to clarify whether the correspondence between the dominant vibrations of the MV and the pulsation of the heart can be demonstrated or not in patients with complete $\mathrm{A}-\mathrm{V}$ block, in which the ventricles beat at very slow rate.

Received for publication, July 19, 1969. 
The MV of the thenar was recorded by means of a MV recording technique ${ }^{3}$ in normal young adults and patients with complete A-V block, who sometimes presented milder symptoms of dizziness and faintness at rest. In addition to the MV, the EKG was also traced simultaneously to elucidate the MV related to the heartbeat. Figure 1 shows the MV and EKG tracings of a normal subject (A) and a patient with complete $A-V$ block $(B)$ in the relaxed waking state, respectively. The dominant vibrations of the MV were observed to be mainly made up of two definite groups of vibrations corresponding to $R$ and $T$ waves of $E K G$ in the patient with complete A-V block as well as in the normal subject, although the intervals between the dominant vibrations of each group also prolonged according to the marked lengthening of the $R-R$ interval in the EKG. The correspondence between the MV and EKG was recognized to be more evident in complete A-V block than in normal cases probably because of marked bradycardia in the former. Furthermore, the correspondence between the MV and $\mathrm{P}$ waves in the EKG was also observed slightly in complete A-V block, in which it was detected in the EKG for the atria to beat independently at the normal sinus rate. The existence of the MV components corresponding to the $R$ and $T$ waves of the EKG shows that there are two groups of vibrations in the MV corresponding to the first and second components of the heartbeat or the systolic and diastolic waves of the BCG. The MV components corresonding to the $\mathrm{P}$ wave in the $\mathrm{EKG}$, on the other hand, suggest the occurrence of mechanical vibrations over the body surface related to
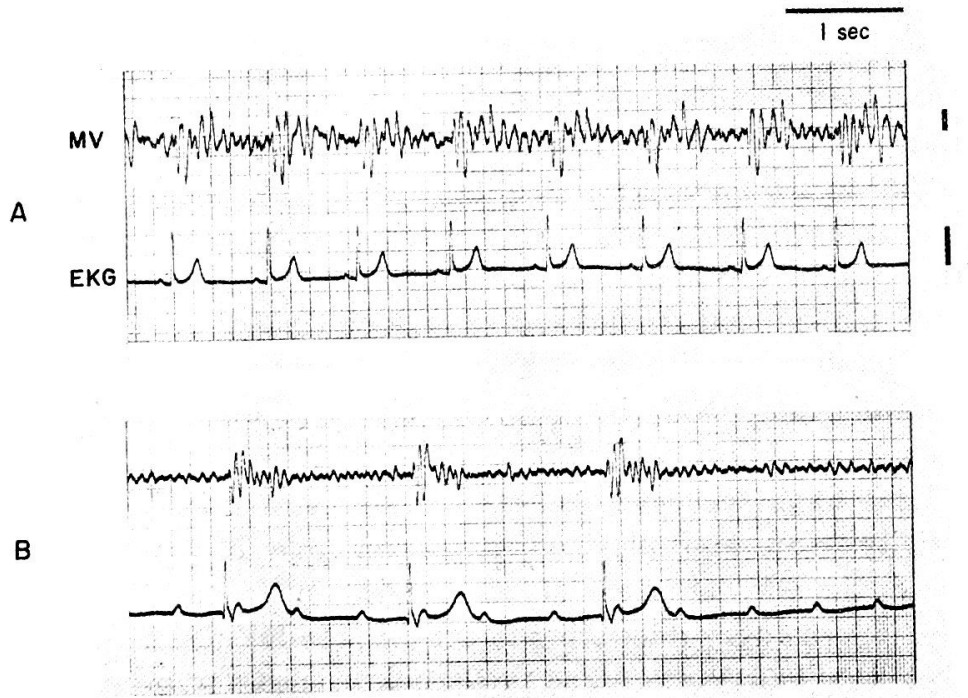

Fig. 1. Microvibration (MV, left thenar) and electrocardiogram (EKG, Lead II) in a normal 23-year-old female(A). and a 15-year-old female with complete A-V block(B). Each calibration in the right side indicates $1 \mathrm{mV}$ in both $\mathrm{MV}$ and EKG. Note marked lengthening of the R-R interval in the EKG and the appearance of the dominant components of the MV corresponding to the $R$ and $T$ waves in the EKG of a patient with complete A-V block. 
the atrial systole, which are usually very difficult to be detected because of a group of vibrations of low amplitude.

From the above-mentioned results, it seems likely that the dominant vibrations of the MV over the human body surface consist of ballistocardiographic components at rest.

\section{Acknowledgment}

We are indebted to our fellow researchers of the Department of Pediatrics, Hirosaki University School of Medicine, for their courtesy in the examination of the subjects.

\section{References}

1) Inanaga, K. On the minor tremor over the human body surface. Clin. Electroencephal. (Jap.), 1959, 2, 127-134.

2) Sugano, H. \& Inanaga, K. Studies on minor tremor. Jap. J. Physiol., 1960, 10, 246257.

3) Ozaki, T., Sato, K., Awazu, T., Mimura, K., Honda, N., Teramoto, S. \& Kitajima, K. Some observations on minor tremors related to heart beat. Jap. J. Physiol., 1962, 12, $484-493$.

4) Ozaki, T., Yamamoto, M. \& Fujiwara, K. On the relationships between minor tremor and ballistocardiogram in man. Acta med. Nagasaki, 1965, 9, 120-134.

5) Sasaki, S., Takahashi, H., Fujiwara, K. \& Ozaki, T. On the effect of eardioinhibitory refexes on the microvibration on body surface. J. Physiol. Soc. Jap. (Jap.), 1969, 13, $288-289$.

6) Buskirk, C.V. \& Fink, R.A. Physiologic tremor. Neurology, 1962, 12, 361-370.

7) Yap. C.B. \& Boshes, B. The frequency and pattern of normal tremor. Electroenceph. clin. Neurophysiol., 1967, 22, 197-203.

8) Yoshii, N., Inaba, E. \& Arai, S. Experimental studies on the minor tremor of body surface in dogs and rabbits. Med. J. Osaka Univ., 1965, 15, 345-358. 